\title{
Large-Scale Distributed Photovoltaic Power Dispatching and Operation Management Review
}

\author{
Nan Zhang1, Yuefeng Wang1, Yuehui Huang1, Dewei Liu'1, Yunfeng Gao', Haifeng Li² \\ ${ }^{1}$ Renewable Energy Department, China Electric Power Research Institute, Beijing, China \\ ${ }^{2}$ State Grid Jiangsu Electric Power Company, Nanjing, China \\ Email: zhangnan@epri.sgcc.com.cn
}

Received January 2015

\begin{abstract}
Distributed photovoltaic power (PV) is the main development model of distributed generation. It is necessary to research on dispatching and operation management with large-scale distributed PV connected. This paper analyzes development status, technical requirement and dispatching and operation management situation of distributed PV in Germany and China. Then introduce the preparation of distributed PV dispatching and operation management criterion. Through summarizing the experiences and lessons of large-scale distributed PV development in Germany, it gives advice to the development of distributed PV dispatching and operation management in China.
\end{abstract}

\section{Keywords}

Distributed Photovoltaic Power, Grid-Connected, Dispatching and Operation, Priority Consumption

\section{Introduction}

With the national energy policy, distributed generators (DG) develop rapidly in China. It is estimated that the capacity of distributed wind power, distributed photovoltaic power (PV) and gas will be $20 \mathrm{GW}, 60 \mathrm{GW}$ and 50 GW respectively in 2020. The rapid development will bring new challenges to the grid safety, power quality and economical operation [1]. Basing on the domestic actual situation, distributed photovoltaic power (PV) is the main development model of distributed generation. In order to master the problem of dispatching and operation with PV accessed in advance, it is necessary to research on dispatching and operation management with largescale distributed PV accessed.

Distributed PV in China is developed late, so using the experience and lesson of other countries for reference is necessary. Distributed PV is developed fast in Germany. According to some statistics, energy output of PV in Germany reach to $20 \mathrm{GW}$ in 2012, which is 4.5 percent of all types power sources' energy output [2]. In this paper, firstly, the current situation, technical requirements and operation management criterion of distributed PV in Germany is analyzed. Secondly, technical requirements and operation management criterion of distributed PV 
in China is analyzed. Lastly, according to the experience and lesson of large-scale distributed PV development in Germany, some suggestion about distributed PV dispatching and operation management in China is proposed.

\section{Distributed PV Dispatching and Operation Management in Germany}

\subsection{Current Dispatching and Operation Management Situation in Germany}

The Germanic renewable energy law which revised in 2004 encouraged private PV installation. Since then, explosion of PV capacity begin as shown in Figure 1.

Generally speaking, Germanic PV connected to medium-low voltage grid. As of the end of 2012, 99.5 percent of Germanic PV connected to grid below $60 \mathrm{kV}$, in which 65 percent PV connected to 380/220 V grid as shown in Figure 2.

Two criterions aiming at distributed PV which connected to grid below $60 \mathrm{kV}$ are proposed in Germany. The criterion name is "Technical minimum requirements for the connection to and parallel operation with low-voltage distribution networks" (below $1 \mathrm{kV}$ ) [3], "technical minimum requirements for the connection to and parallel operation with medium-voltage distribution networks" (between $1 \mathrm{kV}$ to $60 \mathrm{kV}$ ) [4]. The criterions stipulate technical requirements of distributed generation connection, and management requirement of connection, debugging, acceptance, operation and protection in detail.

"Technical minimum requirements for the connection to and parallel operation with medium-voltage distribution networks" summarize the basic requirement of power sources connected to medium-voltage distribution networks. The criterion which started in 1 Jan. 2009 apply to plan, construction, operation and modification of power sources as hydropower, PV, wind power and other distributed generation.

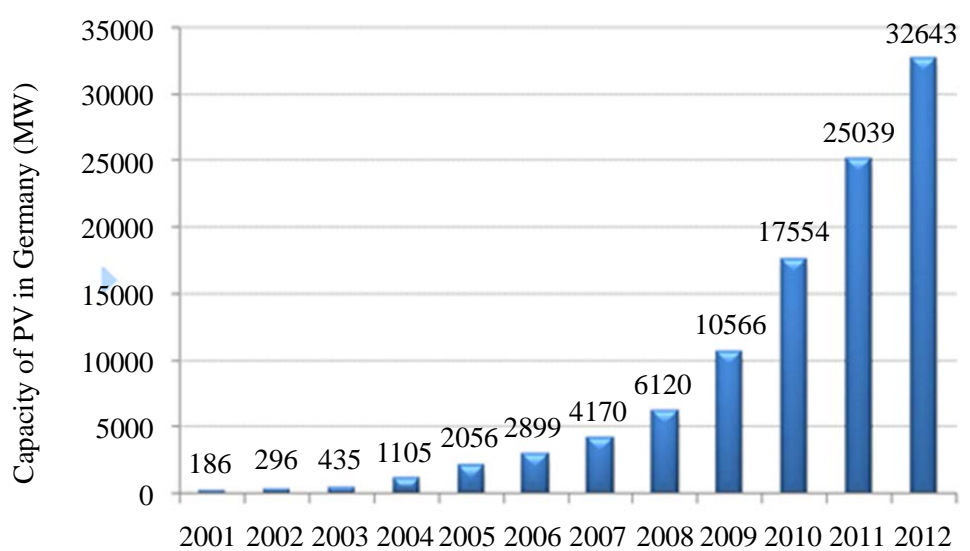

Figure 1. Growth trend of PV installed capacity in Germany.

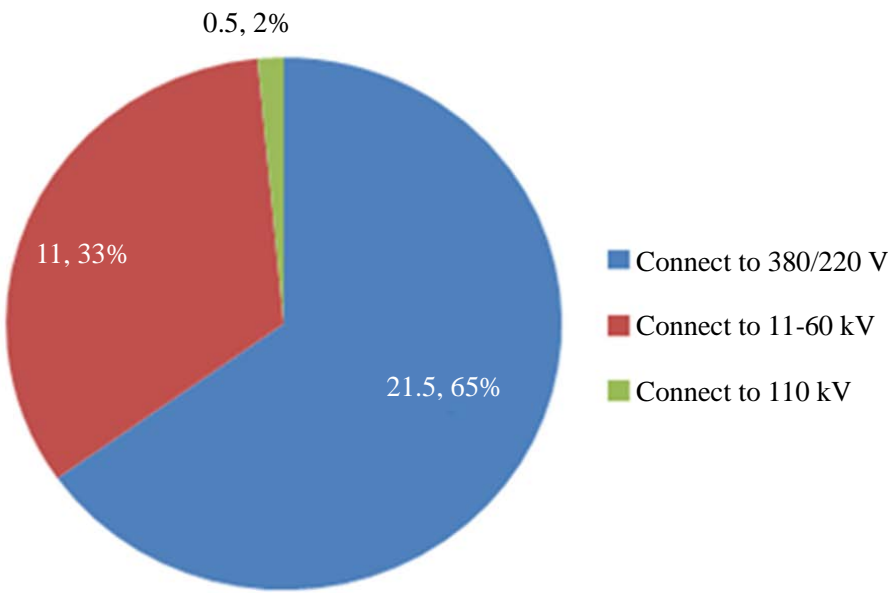

Figure 2. Grid-connected PV in Germany (GW). 
"Technical minimum requirements for the connection to and parallel operation with low-voltage distribution networks” (VDE-AR-N 4105) is drafted by verband Deutscher Elektrotechnikere. v (VDE). It is started in 1 Jul. 2012. VDE-AR-N 4105 proposes DG management requirement contains regulations, application document, and so on. The criterion apply to power sources as hydropower, PV, wind power and other distributed generation which connected to low-voltage distribution networks below $1 \mathrm{kV}$.

\subsection{Current Problem of Distributed PV in Germany}

There are so many problems in the process of distributed PV development. Following are the main points.

Firstly, unit capacity of distributed PV is small and its amount is huge. In 2010 and 2011, the amount of newly installed PV system below $50 \mathrm{kWp}$ was 446.7 thousand, which is 92 percent of newly installed PV system amount. The home-basic distributed PV is raising the proportion more and more in the recent years, as shown in Figure 3.

Secondly, the information of distributed PV could not be real time monitored. Before 2012, distributed PV above $100 \mathrm{~kW}$ is required to be monitored in Germany and the requirement is changed to $50 \mathrm{kWp}$ after 2012. Distributed PV monitoring information transmits to distribution network operators. The information is submitted to transmition grid operators at regular intervals. It is difficult to get real-time output of distributed generation. This brings many difficulties to dispatching.

Thirdly, technology reform of a large amount home-basic distributed PV is difficult, the grid operation will be risky in the future. Laws and regulations are updated to reinforce distributed PV management in Germany. However, the development velocity of distributed PV was underestimated in the initial stage, which leading to a large amount home-basic distributed PV unable to be reformed. In the future, Germany grid must face higher operation cost and bigger operation risk.

\section{Distributed PV Dispatching and Operation Management in China}

\subsection{Current Dispatching and Operation Management Situation in China}

In recent years, because of lower PV subsidies abroad, the problem of PV excess capacity is serious in China. In order to promote domestic PV development, PV installed capacity increases substantially. As shown in Figure 4, the annual growth rate of PV capacity during 2004 to 2010 is 62 percent, the growth is quicker since 2011. It is estimated that PV capacity of China will be 21 GW in 2015.

\section{Systems $\leq 50 \mathrm{kWp}$ are dominating the market with $37 \%$ of capacity}

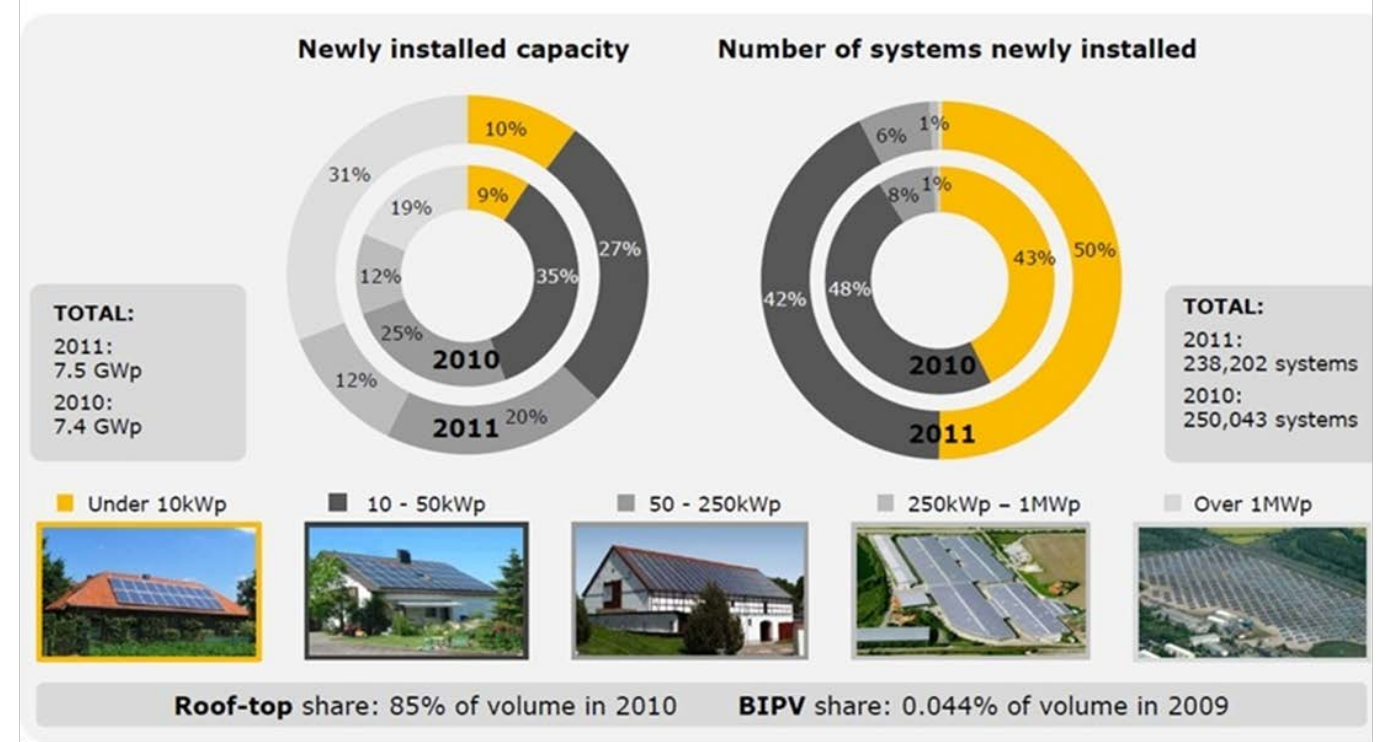

Figure 3. Added PV installed capacity and unit of Germany in 2010 and 2011. 


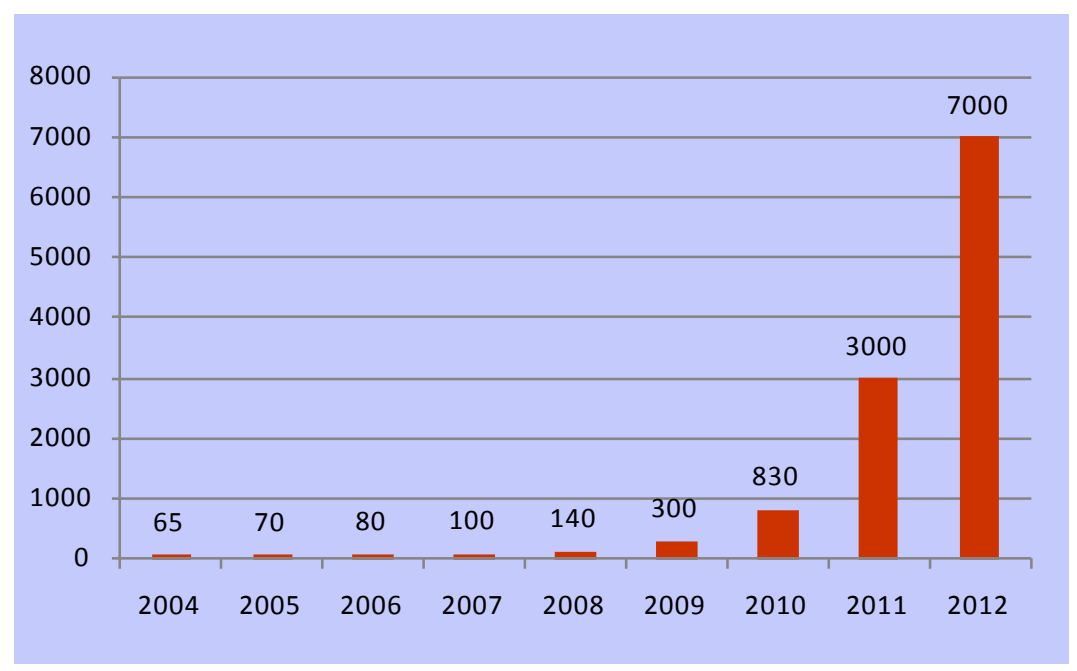

Figure 4. PV installed capacity in China (MW).

In the respect of distributed PV operation management requirements, State Grid Corporation of China (SGCC) issued a document called "SGCC notification about issuing distributed PV connection related advice and standard (revised)" (SGCC (2013) NO.1781) [5]. The document apply to distributed PV that connected to grid below $10 \mathrm{kV}$ and total capacity of a point of common coupling is less than $6 \mathrm{MW}$. The document involves distributed PV connection application, connection project design, commissioning acceptance, operation and other technical steps.

National Energy Administration issued "Interim Provisions for distributed PV project” [6]. The provisions required "power enterprises should build comprehensive technical system combined distributed PV operation monitoring, power forecast and optimizing operation in the area with large-scale distributed PV".

\subsection{Current Problem of Distributed PV in China}

Comparing with experience and lesson of Germany, there are some management problem exists.

Firstly, there is some hidden danger which could cause danger for grid operation. The No.1781 document of SGCC stipulates that sales department taking the charge of review and approval connection scheme. Scheme confirmation sheet is not submitted to dispatching department. In the step of acceptance, sales department is responsible for acceptance. In the step of operation, generating equipment send energy information to sales department once a day. According to lessons of Germany, once getting support of policy and finance, the amount of distributed PV connected to 380/220V grid will be soared. As a result, Grid corporation should audit parameter that involving grid safe [7] [8].

Secondly, real-time information of distributed PV which connected with 220/380V grid could not be monitored. It could cause danger for grid operation. The No.1781 document of SGCC stipulates that distributed PV which connected with 220/380V grid with no need to submit installed capacity, generator type and other key information to dispatching department. According to lessons of Germany, if dispatching department could not have the real-time information of distributed PV that connected with 220/380V grid, the grid operation will be in danger once the amount of distributed is soared [9] [10].

Thirdly, there is no technology support system for distributed PV at present. We have not built distributed generation monitoring system, forecasting system and dispatching automation system. The No.1781 document of SGCC stipulates that distributed generation information could transmit by outer net. However, how to collect and transmit is gap. It is necessary to build monitoring system, forecasting system and dispatching automation system. Functional specification and design of the system is an urgent need [10] [11].

\section{Suggestion of Distributed PV Dispatching and Operation Management in China}

In view of experience and lesson of Germany, and our actual situation, this paper proposes suggestion in the step of connection management, operation management, overhaul management and so on. 


\subsection{Connection Management Step}

After confirming system connection scheme, power equipment information, power equipment geographical location and system connection scheme should be submitted to dispatching department. Dispatching department should participate in commissioning and checking of distributed PV which connected with 220/380V grid.

\subsection{Operation Management Step}

In the respect of monitoring, distributed PV monitoring system should be built, which could monitor distributed PV real time output data every 15 minutes. In the respect of power forecasting, distributed generation forecast system should be built at scheduling department at the provincial level. The system could do short-term and ultra-short term forecasting for distributed PV connected to $10 \mathrm{kV}$ or $380 / 220 \mathrm{~V}$ grid. In the respect of operation and control, distributed PV owner should assign equipment operators to be responsible for distributed PV operation.

\subsection{Overhaul Management Step}

The distributed PV that connected to $10 \mathrm{kV}$ grid should be overhauled as power source. It is managed by scheduling department. The distributed PV that connected to 380/220V grid should be managed by sales department. The overhaul scheme should be submitted to scheduling department in advance.

\subsection{Professional Management Step}

Scheduling department is responsible for protection, automatic safety device, communication and other professional management step. The distributed PV that connected to 380/220V grid should pick-up operation information in real time and send the information to dispatching automation system, which contains generation information per 15 minutes.

Since distributed PV is dispersed in so many spots and quite wide areas, the common distributed PV owner do not have the capabilities to forecast power output. Grid corporation should be responsible for forecasting. This paper proposes that building gridding forecasting system at scheduling department of the provincial level and city level. Figure 5 shows gridding forecast sketch.

Monitoring distributed PV uses gridding management at the same time. The gridding information goes through outer net transmission, aggregation and fusion, passes isolating device for security and accesses to distributed PV forecasting and monitoring module of scheduling department. Figure 6 shows framework of distributed PV gridding monitoring system.

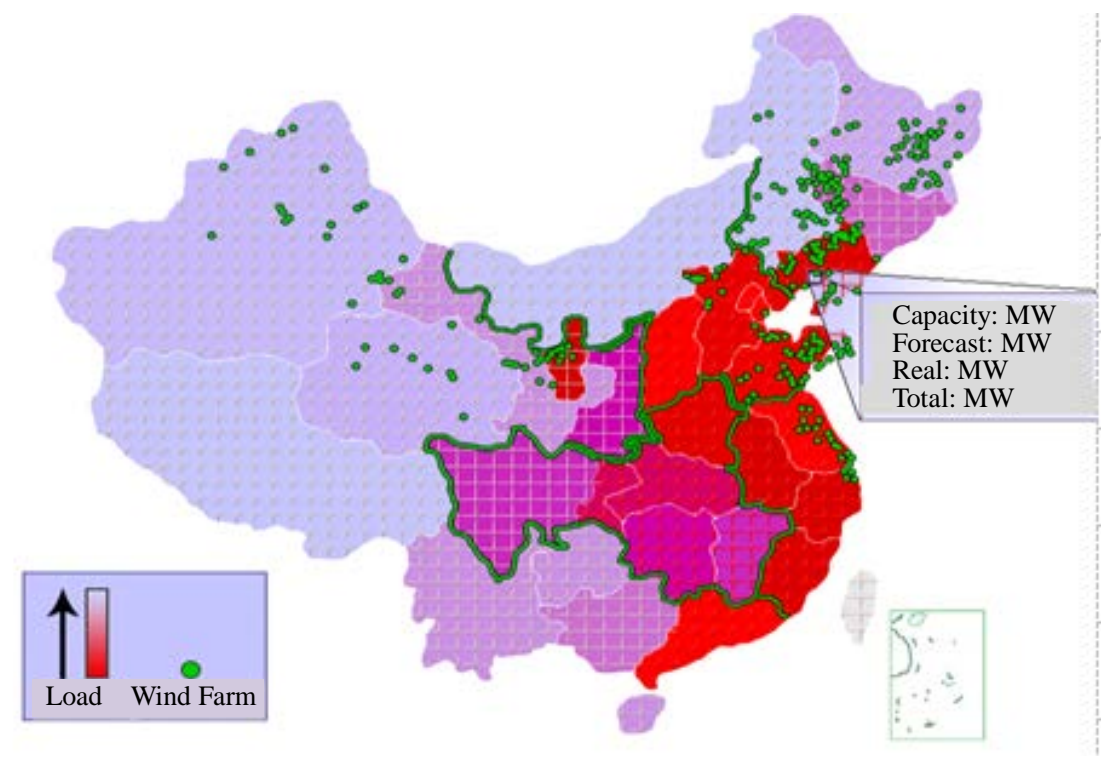

Figure 5. Gridding forecast sketch. 


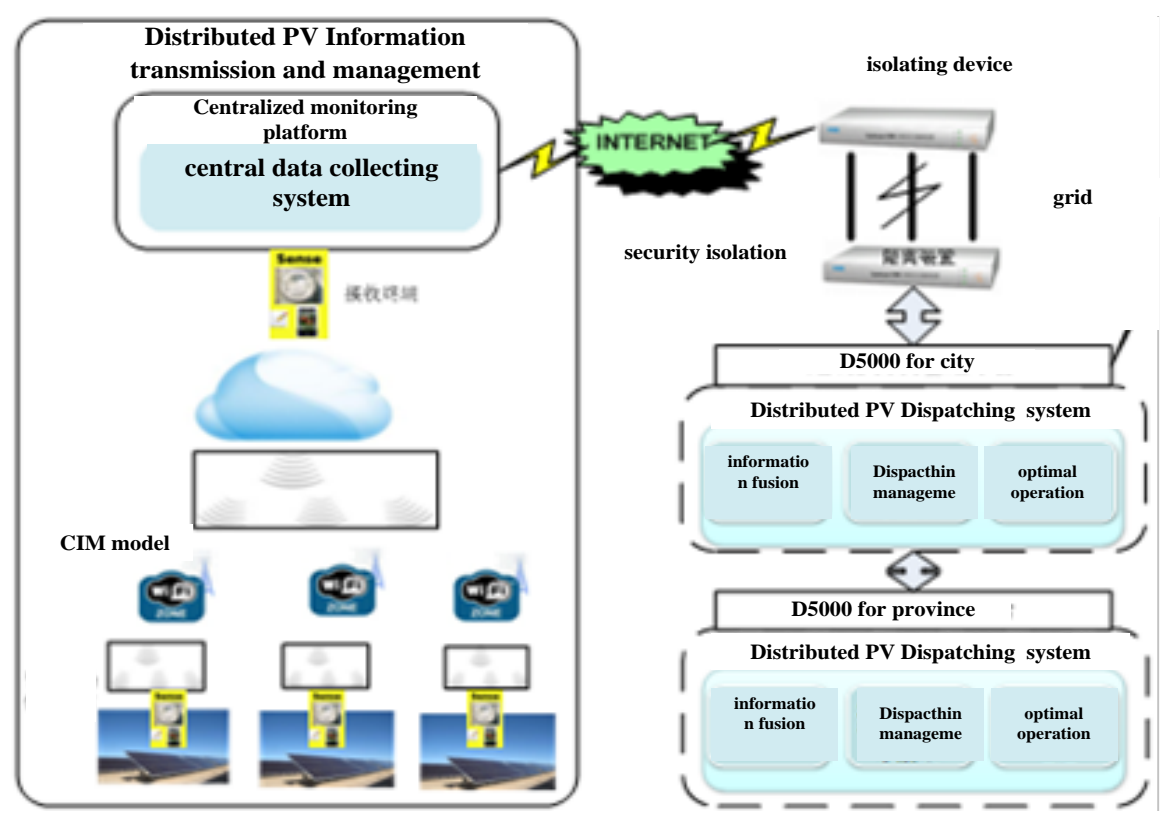

Figure 6. Gridding monitoring system frame of distributed PV.

\section{Conclusion}

Current situation of distributed PV in Germany and China is compared in this paper. Besides, experience and lesson of Germany is analyzed. On this basis, this paper proposes management suggestion in the step of distributed PV connection. Research result of this paper helps to realize large-scale priority integration and scientific management of distributed PV.

\section{Funding}

This work was supported by the State Grid Corporation Science and Technology Project.

\section{References}

[1] IEEE1547'M (2003) IEEE Standard for Interconnecting Distributed Resources with Electric Power Systems.

[2] Gu, Y.G., Wang, K. and Zhang, B. (2010) Distributed Generation Technologies and Their Current Applications. Power System and Clean Energy, 26, 38-43.

[3] (2009) Technical Minimum Requirements for the Connection to and Parallel Operation with Medium-Voltage Distribution Networks.

[4] VDE-AR-N 4105 (2012) Technical Minimum Requirements for the Connection to and Parallel Operation with LowVoltage Distribution Networks.

[5] (2013) SGCC Notification about Issuing Distributed PV Connection Related Advice and Standard (Revised).

[6] (2013) Interim Provisions for Distributed PV Project.

[7] (2013) Interim Provisions for Distributed Generation Project.

[8] Wei, X.X. and Liu, S.W. (2010) Analysis on International Development in Distributed Generation and Its Revelation to China. Energy Technology and Economics, 22, 58-65.

[9] Brandstätt, C., Brunekreeft, G. and Friedrichsen, N. (2011) Locational Distribution Network Pricing in Germany. IEEE PES GM, Detroit, 28 July 2011. http://dx.doi.org/10.1109/PES.2011.6039320

[10] (2012) Distributed Generation Connection Situation Research Report. State Grid Energy Research Institution.

[11] Gonzalez-Longatt, F. and Fortoul, C. (2008) Review of the Distributed Generation Concept: Attempt of Unification. Universidad Central de Venezuela. 